\title{
Strains of scattering of near-field of a point source
}

\author{
M D SHARMA \\ Department of Mathematics, Kurukshetra University, India 136119. \\ e-mail: mohan_here@rediffmail.com
}

Three dimensional scattering of near-field is studied for dilatation and rotation in the time domain. The perturbation method is applied to solve the equation of motion for the first order scattering from a weak inhomogeneity in an otherwise homogeneous medium. The inhomogeneity is assumed close enough to the point source so that the near-field intermediate wave is dominating over the far-field spherical $P$ and $S$ pulses. The integral expressions are derived to relate dilatation and rotation of scattering to the radial fluctuations of velocities and density in the inhomogeneity. These integrals are solved to calculate the strains of scattering from (a part of) an inhomogeneous spherical shell of arbitrary curvature. Variable curvature may allow the shape of inhomogeneity volume element to change uniformly from spherical to rectangular. Rotation of scattering from a spherical shell is independent of $P$ wave velocity inhomogeneity. Dilatation of scattering does not involve $S$ wave velocity inhomogeneity but its gradient. The back scattering results are obtained as a special case. Strains are computed numerically, for hypothetical models to study the effects of various parameters viz., velocity inhomogeneity, distance of source from inhomogeneity and from receiver, and thickness of inhomogeneity. The curvature of the spherical shell is varied to study the effects of the shape of inhomogeneous volume element on scattering.

\section{Introduction}

One of the major goals of seismology is to develop a constitutive law that characterises the material response to an applied stress. Such a law would help to discover the stress conditions on faults before and during earthquakes. The detailed measurements of temporal and spatial variations of near-fault crustal deformations may explain the material response to the stress changes there. Routine observations of tilt and strain around an active fault is important for short-term earthquake prediction. Rikitake (1981) asserts that 'crustal deformation may be the most useful phenomenon for specific earthquake prediction'. The idea behind this is that whatever the nature of the earthquake preparatory process, it should be some type of mechanical process which would have to be accompanied by crustal movements. Elastic wave scattering could be an effective tool to monitor the strain/stress changes at any fault in the earth's crust. The study of dynamism at such a fault may help in improving the monitoring techniques, being used presently. This requires an advancement of scattering theory by incorporating the various aspects of rock property distributions in the theoretical models.

The basis for any advanced scattering theory is the elastic wave scattering by a single inclusion in a homogeneous elastic medium. Korneev and Johnson (1993) gave the compact solutions for single scattering by spheres of arbitrary contrast and tested them numerically. In a related work, Gritto et al (1995) have tested the validity of the commonly used far-field approximation. The absence of a magic method that can solve a difficult scattering problem, resulted in a good number of different approaches. In the approach which best serves the purpose (Leary 1995), the wave equation is written in terms of a uniform average medium about

Keywords. Scattering; volume inhomogeneity; near-field; point source; strains.

Proc. Indian Acad. Sci. (Earth Planet. Sci.), 113, No. 2, June 2004, pp. 247-257 
which the heterogeneities are local fluctuations. A first order perturbation approximation is used to express the coda wave-field in terms of scattering by a primary wavelet interacting with heterogeneities (Aki and Richards 1980). Typical fluctuation of around 5 per cent in crustal velocity (Leary 1990) favours the first order perturbation approach in solving the elastic wave equation for a heterogeneous medium. Leary and Abercrombie (1994) have shown that first order scattering alone can be sufficient to model coda energy up to 20s. Moreover, if source and receiver could be placed near to the inhomogeneity, the first order scattering may represent the propagation regime more effectively.

The factors affecting the ground motion are source effects, wave path effects and site response. The complex effects of wave paths can be ignored in the near-field studies. Hence, the data from nearfield offer better information on the parameters of the source in a nearby fault zone. Precursory phenomena of an expected earthquake are related to near-source changes in shear and dilatational strains within the fault zone and in the bordering region. The seismic data used in earthquake engineering are collected in the near-field. The stress related dynamism around an active fault is also a near-field effect. Characterisation of fractured heterogeneous reservoirs for oil-water substitution events and for fluid transport phenomena is another important field where near-field of source cannot be neglected. However, the scattering studies available in the literature, viz., Sato (1984); Wu and Aki (1985a,b, 1988); Wu (1989); Rose (1989); Leary (1990, 1995); Gurevich et al (1992, 1998); Sharma (2001), assume the point source to be either a $P$ wave source or an $S$ wave source. Hence, the primary waves radiating from the source carry only the far-field response of a point source. Not a single study could be found which studied the scattering of the near-field of a point source.

The strainmeter is one of the basic instruments used to measure the deformation of a small part of the earth. Borehole strainmeters are commonly used for earthquake prediction programs in some regions of Japan. Keeping in mind the strainmeter arrays installed along active faults, the study of strains of near-field scattering may be useful for simulation purposes. Moreover, the scattering studies available in literature calculate the displacement of scattered phases assuming that both the source and receiver are far from the inhomogeneity. The author, here, solves the integral expression for dilatation and rotation of scattering, for the near-field term of Green's function (Aki and Richards 1980) from an impulsive point source. Receiver location is arbitrary. When the point of source is near to the inhomogeneity, the work presented represents the ever ignored part of scattering studies. For arbitrary position of source, this study provides a correction supplement to the conventional studies of scattering of far-field from a point source.

\section{First order scattering strains}

A finite volume weak inhomogeneity is considered in an isotropic elastic homogeneous medium of wave velocities $\alpha_{o}, \beta_{o}$ and density $\rho_{o}$. Wave velocities and density of this inhomogeneity are defined as

$$
p=p_{o}+\delta p ;(p=\alpha, \beta, \rho),
$$

where $\delta p\left(|\delta p| \ll\left|p_{o}\right|\right)$, denote the small perturbations in velocities and density. The displacement in the inhomogeneous medium is written as $\mathbf{u}=\mathbf{u}^{o}+\delta \mathbf{u}$, which is a sum of 'primary waves' $\mathbf{u}^{o}$ and 'scattered waves' $\delta \mathbf{u}$. Inhomogeneity is weak so that $|\delta \mathbf{u}| \ll\left|\mathbf{u}^{\circ}\right|$, and, hence, scattered waves obey the wave equations for the unperturbed medium.

Following Aki and Richards (1980), dilatation and rotation for the first order scattering of waves from a small volume $V$ and recorded at $\mathbf{r}$, are written as

$$
\begin{aligned}
\nabla \cdot \delta \mathbf{u}(\mathbf{r}, t)= & \frac{1}{4 \pi \alpha_{o}^{2}} \int \frac{1}{|\mathbf{r}-\mathbf{q}|} \nabla \cdot \mathbf{Q} \\
& \times\left(\mathbf{q}, t-\frac{|\mathbf{r}-\mathbf{q}|}{\alpha_{o}}\right) d V(\mathbf{q}), \\
\nabla \wedge \delta \mathbf{u}(\mathbf{r}, t)= & \frac{1}{4 \pi \beta_{o}^{2}} \int \frac{1}{|\mathbf{r}-\mathbf{q}|} \nabla \wedge \mathbf{Q} \\
& \times\left(\mathbf{q}, t-\frac{|\mathbf{r}-\mathbf{q}|}{\beta_{o}}\right) d V(\mathbf{q}) .
\end{aligned}
$$

The body force $\mathbf{Q}$, arises from the interaction of inhomogeneity with the primary waves from source. It vanishes outside the volume of inhomogeneity $V$. Following Sharma (2001), the $\mathbf{Q}$ is expressed as

$$
\begin{aligned}
Q_{i}= & \left(2 \alpha_{o} \delta \alpha\right) u_{j, i j}^{o}+\left(2 \alpha_{o} \delta \alpha+\alpha_{o}^{2} \frac{\delta \rho}{\rho_{o}}\right)_{, i} u_{j, j}^{o} \\
& +\left(2 \beta_{o} \delta \beta\right)\left(u_{i, j j}^{o}-u_{j, i j}^{o}\right) \\
& +\left(2 \beta_{o} \delta \beta+\beta_{o}^{2} \frac{\delta \rho}{\rho_{o}}\right)_{, j}\left(u_{i, j}^{o}+u_{j, i}^{o}-2 \delta_{i j} u_{k, k}^{o}\right) .
\end{aligned}
$$




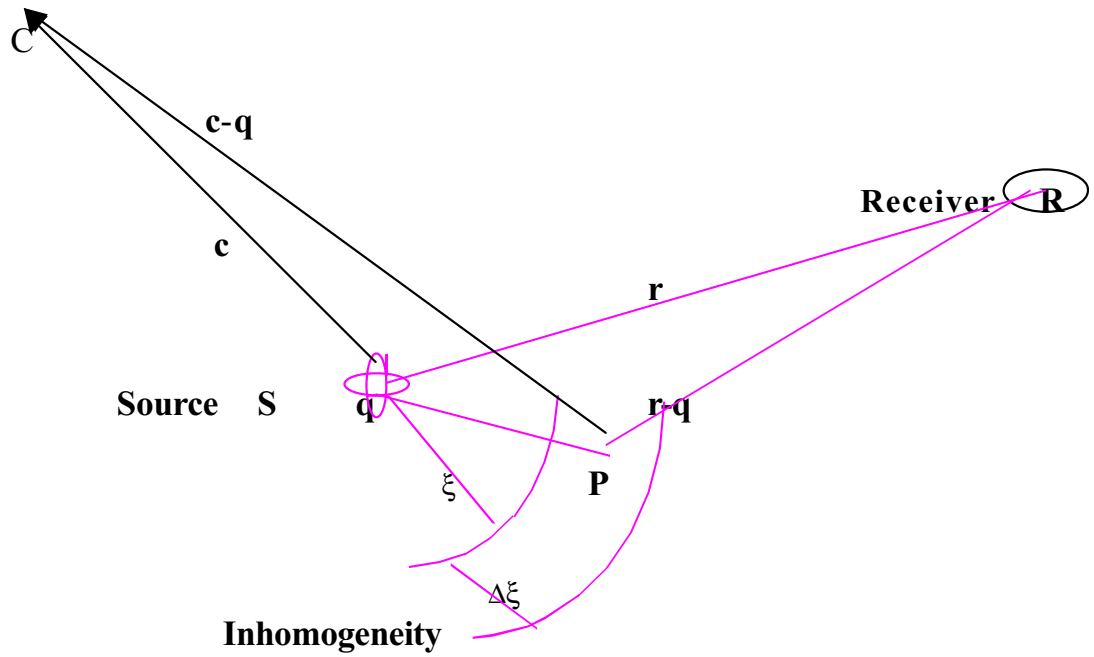

Figure 1. Geometry of the medium.

\section{Geometry of the medium: (figure 1)}

Consider a Cartesian co-ordinate system with source placed at $S \leftrightarrow\left(S_{x}, S_{y}, S_{z}\right)$. The unit vector $\mathbf{n}=\left(n_{x}, n_{y}, n_{z}\right)$ defines the direction of the force at $S$. An arbitrary location for receiver is represented by $R \leftrightarrow\left(R_{x}, R_{y}, R_{z}\right)$. Hence, we have $\mathbf{r}=\left(R_{x}-S_{x}, R_{y}-S_{y}, R_{z}-S_{z}\right)$. An arbitrary point $P \leftrightarrow\left(P_{x}, P_{y}, P_{z}\right)$ in the inhomogeneity, is represented by the variable vector $\mathbf{q}=\left(P_{x}-S_{x}, P_{y}-\right.$ $S_{y}, P_{z}-S_{z}$ ) from source. A spherical shell inhomogeneity of thickness $\Delta \xi$ is centred at a point $C$ at a distance $c$ from source $S$. Then $C$ is represented by $\mathbf{c}=\left(C_{x}-S_{x}, C_{y}-S_{y}, C_{z}-S_{z}\right)$. The source $S$ is at a radial distance of $\xi$ from the inner edge of shell. The change in the value of $c$ changes the curvature of the inhomogeneity and hence its shape with respect to source. For greater values of $c$ the spherical shell shapes into a rectangular volume element. Denote $\zeta=|\mathbf{q}-\mathbf{c}| \cdot d V=\zeta^{2} d \zeta d \Omega ; d \Omega=d \chi d \phi$ represents the solid angle subtended by the inhomogeneity at $C$. $\zeta$ will be varying from $c+\xi$ to $c+\xi+\Delta \xi$. For a complete spherical shell, $\chi(=\cos \theta)$ varies from -1 to 1 and $\phi$ varies from 0 to $2 \pi$.

In a spherical co-ordinate system centred at $C$ with $-\mathbf{c}$ being its polar axis, the variable $\mathbf{q}=$ $(q, \theta, \phi)$ is related to its Cartesian components by $q_{x}=\zeta \sqrt{1-\chi^{2}} \cos \phi, q_{y}=\zeta \sqrt{1-\chi^{2}} \sin \phi$ and $q_{z}=\zeta \chi-c$.

\section{Strains of scattered near-field}

The sources of small earthquakes and artificial sources can be approximated as point sources. Green's function representation of radiation from a point source is the basic technique used for simulation studies. Green's functions are convoluted with the source time function to calculate the ground motion at a site. Studies available in the literature use impulsive point source as the generator of either $P$ waves or $S$ waves, which represent only the far-field of a point source. Heaviside time function term representing an intermediate wave and more effective near the source is always ignored.

Faulting is considered to be the process of failure in shear. To understand the physical processes actually occurring in the source region, one must examine the way in which the material failure nucleates and spreads. Information about a seismic source is contained in seismic pulse shape also called source time function. The source parameters, slip, stress changes on the faults surface are assumed to be similar to a step or Heaviside time function. Nucleation of crack near an existing fault starts with a slippage at the fault which is represented by a jump discontinuity rather than a symmetric impulsive form. All these factors compel us not to abandon the part of point source radiation represented by a Heaviside time function, i.e., nearfield term.

According to Aki and Richards (1980), at the distance of $(2 \pi)^{-1}$ times of wavelength the nearfield and far-field of a point source radiation are the same. This implies that when an inhomogeneity is near to the source (e.g., advancement of oil-water barrier in a producing reservoir, flaw detection through non-destructive testing, fluidtransport imaging in fractured gas reservoirs) or when to study the propagation of waves with large wavelength (e.g., seismic waves) near-field cannot be ignored. Not only does the data from the nearfield offer information on the source parameters unattainable from far-field alone, but the wave propagation studies of the near-field may provide us with the ability of predicting the seismic motion 
due to an expected earthquake in a nearby fault zone.

The source at $S$ is an impulsive point unit force in the direction $\hat{\mathbf{n}}$. The radially polarised near-field from an impulsive point source, given by,

$\mathbf{u}^{o}(\mathbf{q}, t)=4 \pi u_{o} \mathbf{d} \frac{s_{o}^{2} \alpha_{o}}{q^{3}} t\left[H\left(t-\frac{q}{\alpha_{o}}\right)-H\left(t-\frac{q}{\beta_{o}}\right)\right]$,

represents the displacement at a point $\mathbf{q}$ in the near-field of source. Following Aki and Richards (1980), it is the near-field part of the radiation from an impulsive point unit force. The normalisation of equation (5) is the displacement $4 \pi u_{o}$ at a distance $s_{o}$ from the source and at time $t=s_{o} / \alpha_{o}$. The polarisation vector $\mathbf{d}$ is given by: $d_{i}=\left(3 \gamma_{i} \gamma_{j}-\delta_{i j}\right) n_{j}$, where, direction cosines $\gamma_{j}=q_{j} / q ;(j=x, y, z)$, control the angular dependence of radiation.

The near-field motion, given by equation (5), acts as primary wave which after interaction with inhomogeneity generates a scattering source in the form of body force $\mathbf{Q}$. The scattered waves from such a source have their strains defined by the equations (2) and (3). These integral expressions calculate the strains of scattered near-field, to be recorded at a receiver at $R$. The velocity and density perturbations, are represented by $F(=$ $\left.\frac{\delta \alpha}{\alpha_{o}}\right), G\left(=\frac{\delta \beta}{\beta_{o}}\right)$, and $E\left(=\frac{\delta \rho}{\rho_{o}}\right)$. These are assumed to be twice differentiable functions of $q$. Solving the expressions (2) and (3), the dilatation $(\nabla \cdot \delta \mathbf{u})$ and rotation $(\nabla \wedge \delta \mathbf{u})$ of scattering are obtained as follows:

$$
\nabla \cdot \delta \mathbf{u}(\mathbf{r}, t)=u_{o} \alpha_{o} s_{o}^{2} \int \chi_{f} X(q) \frac{1}{q^{3}} D\left(t_{d}\right) d V(\mathbf{q}),
$$

$$
\nabla \wedge \delta \mathbf{u}(\mathbf{r}, t)=u_{o} \alpha_{o} s_{o}^{2} \int \hat{\Psi}_{f} X(q) \frac{1}{q^{3}} R\left(t_{r}\right) d V(\mathbf{q})
$$

where, $\chi_{f}=\hat{\mathbf{q}} \cdot \hat{\mathbf{n}}, \hat{\Psi}_{f}=\hat{\mathbf{q}} \wedge \hat{\mathbf{n}}, X(q)=\frac{1}{|\mathbf{r}-\mathbf{q}|}, t_{d}=$ $t-\frac{|\mathbf{r}-\mathbf{q}|}{\alpha_{o}}, t_{r}=t-\frac{|\mathbf{r}-\mathbf{q}|}{\beta_{o}}$.

$D$ and $R$ are given by

$$
\begin{aligned}
D\left(t_{d}\right)= & 4\left[F Y_{q q q}+\left(2 F^{\prime}-\frac{4}{q} F\right) Y_{q q}\right. \\
& \left.+\left(F^{\prime \prime}-\frac{4}{q} F^{\prime}+\frac{4}{q^{2}} F\right) Y_{q}\right] \\
& +2\left[E^{\prime} Y_{q q}+\left(E^{\prime \prime}-\frac{1}{q} E^{\prime}\right) Y_{q}\right]-\left(\frac{\beta_{o}}{\alpha_{o}}\right)^{2}
\end{aligned}
$$

$$
\begin{aligned}
& \times\left[\frac{24}{q}\left\{G^{\prime} Y_{q}+\left(G^{\prime \prime}-\frac{1}{q} G^{\prime}\right) Y\left(q, t_{d}\right)\right\}\right. \\
& \left.+\frac{2}{q}\left\{5 E^{\prime} Y_{q}+6\left(E^{\prime \prime}-\frac{1}{q} E^{\prime}\right) Y\left(q, t_{d}\right)\right\}\right],
\end{aligned}
$$

$$
\begin{aligned}
R\left(t_{r}\right)= & -2\left[G Y_{q q q}+\left(2 G^{\prime}-\frac{4}{q} G\right) Y_{q q}\right. \\
& +\left(G^{\prime \prime}-\frac{10}{q} G^{\prime}+\frac{4}{q^{2}} G\right) Y_{q} \\
& \left.-\frac{6}{q}\left(G^{\prime \prime}-\frac{1}{q} G^{\prime}\right) Y\left(q, t_{r}\right)\right] \\
& -\left[E^{\prime} Y_{q q}+\left(E^{\prime \prime}-\frac{8}{q} E^{\prime}\right) Y_{q}\right. \\
& \left.-\frac{6}{q}\left(E^{\prime \prime}-\frac{1}{q} E^{\prime}\right) Y\left(q, t_{r}\right)\right] \\
& -\left(\frac{\alpha_{o}}{\beta_{o}}\right)^{2} \frac{2}{q} E^{\prime} Y_{q}
\end{aligned}
$$

Subscripts ' $q$ ' to $Y$ denote the derivatives of $Y(q, t)$ with respect to $q$, evaluated at $t=t_{j},(j=d, r)$ where, $Y(q, t)=t\left[H\left(t-\frac{q}{\alpha_{o}}\right)-H\left(t-\frac{q}{\beta_{o}}\right)\right] . H$ is Heaviside unit step function. Primes to $F, G$ and $E$ denote their derivatives with respect to $q$.

\section{Spherical shell inhomogeneity}

To solve the volume integrals in equations (6)-(7), the inhomogeneity is assumed to be a spherical shell of thickness $\Delta \xi$, between two spheres having the same centre at $C$. The approximate $(d \zeta \approx d q)$ volume of the inhomogeneity is given by $d V=$ $\left(c^{2}+q^{2}-2 c q \chi_{c}\right) d q d \chi d \phi . \chi_{c}$ is the cosine of angle between $\mathbf{q}$ and $\mathbf{c}$. Properties of $\delta$ function help to reduce the volume integral to an integral over solid angle $\Omega$ of the inhomogeneity at $C$. The scattering strains are, then, given by

$$
\begin{aligned}
\nabla \cdot \delta \mathbf{u}= & u_{o} s_{o}^{2} \alpha_{o} \int \chi_{f}\left[\frac{v_{2} q_{2}}{\beta_{o}^{2}} D_{1}\left(q_{2}\right)-\frac{v_{1} q_{1}}{\alpha_{o}^{2}} D_{1}\left(q_{1}\right)\right. \\
& \left.+\int_{q_{1}}^{q_{2}} D_{2}(q) d q\right] d \Omega
\end{aligned}
$$


$\nabla \wedge \delta \mathbf{u}=u_{o} s_{o}^{2} \alpha_{o} \int \hat{\Psi}_{f}\left[\frac{v_{2} q_{2}}{\beta_{o}^{2}} R_{1}\left(q_{2}\right)-\frac{v_{1} q_{1}}{\alpha_{o}^{2}} R_{1}\left(q_{1}\right)\right.$

$$
\left.+\int_{q_{1}}^{q_{2}} R_{2}(q) d q\right] d \Omega
$$

$D_{k}$ and $R_{k},(k=1,2)$ are expressed as

$$
\begin{aligned}
& D_{k}(q)=D_{k 2}(q)-2 c \chi_{c} D_{k 1}(q)+c^{2} D_{k 0}(q), \\
& R_{k}(q)=R_{k 2}(q)-2 c \chi_{c} R_{k 1}(q)+c^{2} R_{k 0}(q),
\end{aligned}
$$

where, if we define

$$
\begin{aligned}
v_{1}= & \left|\frac{1}{\alpha_{o}}+X\left(q_{1}\right) \frac{\left(q_{1}-r \chi_{r}\right)}{v_{s}}\right|^{-1}, \\
v_{2}= & \left|\frac{1}{\beta_{o}}+X\left(q_{2}\right) \frac{\left(q_{2}-r \chi_{r}\right)}{v_{s}}\right|^{-1}, \\
q_{o}= & {\left[r \chi_{r}-t v_{s} v_{r}+\left\{r^{2}\left(\chi_{r}^{2}+v_{r}^{2}-1\right)+v_{s}^{2} t^{2}\right.\right.} \\
& \left.\left.-2 r \chi_{r} v_{s} t v_{r}\right\}^{1 / 2}\right] /\left(1-v_{r}^{2}\right), \\
q_{o o}= & .5\left(r^{2}-t^{2} v_{s}^{2}\right) /\left(r \chi_{r}-t v_{s}\right), \\
X_{1}= & -\left(q-r \chi_{r}\right) X(q)^{3}, \\
X_{2}= & -X(q)^{3}+3\left(q-r \chi_{r}\right)^{2} X(q)^{5}, \\
X_{3}= & 9\left(q-r \chi_{r}\right) X(q)^{5}-15\left(q-r \chi_{r}\right)^{3} X(q)^{7},
\end{aligned}
$$

then

$$
\begin{aligned}
D_{12}(q)= & 4\left(\frac{1}{q} X_{2}+\frac{2}{q^{2}} X_{1}-\frac{2}{q^{3}} X\right) F-\left(\frac{\beta_{o}}{\alpha_{o}}\right)^{2} \\
& \times \frac{24}{q^{2}} X G^{\prime}-\left(\frac{2}{q} X_{1}+\left(\frac{\beta_{o}}{\alpha_{o}}\right)^{2} \frac{10}{q^{2}} X\right) E^{\prime},
\end{aligned}
$$

$$
\begin{aligned}
D_{11}(q)= & 4\left(\frac{1}{q^{2}} X_{2}-\frac{2}{q^{4}} X\right) F-\left(\frac{\beta_{o}}{\alpha_{o}}\right)^{2} \frac{24}{q^{3}} X G^{\prime} \\
& -\left\{\frac{2}{q^{2}}\left(X_{1}-\frac{1}{q} X\right)+\left(\frac{\beta_{o}}{\alpha_{o}}\right)^{2} \frac{10}{q^{3}} X\right\} E^{\prime},
\end{aligned}
$$

$$
\begin{aligned}
D_{10}(q)= & 4\left(\frac{1}{q^{3}} X_{2}-\frac{2}{q^{4}} X_{1}\right) F-\left(\frac{\beta_{o}}{\alpha_{o}}\right)^{2} \frac{24}{q^{4}} X G^{\prime} \\
& -\left\{\frac{2}{q^{3}}\left(X_{1}-\frac{2}{q} X\right)+\left(\frac{\beta_{o}}{\alpha_{o}}\right)^{2} \frac{10}{q^{4}} X\right\} E^{\prime},
\end{aligned}
$$

$$
\begin{aligned}
D_{22}(q)= & -t_{d}\left(\frac{\beta_{o}}{\alpha_{o}}\right)^{2} \frac{24}{q^{2}}\left\{2\left(G^{\prime \prime}-\frac{1}{q} G^{\prime}\right)\right. \\
& \left.+\left(E^{\prime \prime}-\frac{1}{q} E^{\prime}\right)\right\} X, \\
D_{21}(q)= & -t_{d}\left(\frac{\beta_{o}}{\alpha_{o}}\right)^{2} \frac{24}{q^{3}}\left\{2\left(G^{\prime \prime}-\frac{1}{q} G^{\prime}\right)\right. \\
& \left.+\left(E^{\prime \prime}-\frac{1}{q} E^{\prime}\right)\right\} X,
\end{aligned}
$$

$$
\begin{aligned}
D_{20}(q)= & -t_{d}\left(\frac{\beta_{o}}{\alpha_{o}}\right)^{2} \frac{24}{q^{4}}\left\{2\left(G^{\prime \prime}-\frac{1}{q} G^{\prime}\right)\right. \\
& \left.+\left(E^{\prime \prime}-\frac{1}{q} E^{\prime}\right)\right\} X,
\end{aligned}
$$

with $v_{s}=\alpha_{o}, v_{r}=\alpha_{o} / \beta_{o}, q_{1}=q_{o o}$ and $q_{2}=q_{o}$; and

$$
\begin{aligned}
R_{12}(q)= & \frac{12}{q^{2}} X G^{\prime}-2\left(\frac{1}{q} X_{2}+\frac{2}{q^{2}} X_{1}-\frac{2}{q^{3}} X\right) G \\
& -\left(\frac{\alpha_{o}}{\beta_{o}}\right)^{2} \frac{2}{q^{2}} X E^{\prime}+\left(\frac{1}{q} X_{1}+\frac{7}{q^{2}} X\right) E^{\prime}
\end{aligned}
$$

$$
\begin{aligned}
R_{11}(q)= & \frac{12}{q^{3}} X G^{\prime}-2\left(\frac{1}{q^{2}} X_{2}-\frac{2}{q^{4}} X\right) G \\
& -\left(\frac{\alpha_{o}}{\beta_{o}}\right)^{2} \frac{2}{q^{3}} X E^{\prime}+\left(\frac{1}{q^{2}} X_{1}+\frac{6}{q^{3}} X\right) E^{\prime},
\end{aligned}
$$

$$
\begin{aligned}
R_{10}(q)= & \frac{12}{q^{4}} X G^{\prime}-2\left(\frac{1}{q^{3}} X_{2}-\frac{2}{q^{4}} X_{1}\right) G \\
& -\left(\frac{\alpha_{o}}{\beta_{o}}\right)^{2} \frac{2}{q^{4}} X E^{\prime}+\left(\frac{1}{q^{3}} X_{1}+\frac{5}{q^{4}} X\right) E^{\prime},
\end{aligned}
$$


$R_{22}(q)=t_{r} \frac{6}{q^{2}}\left\{2\left(G^{\prime \prime}-\frac{1}{q} G^{\prime}\right)+\left(E^{\prime \prime}-\frac{1}{q} E^{\prime}\right)\right\} X$

$R_{21}(q)=t_{r} \frac{6}{q^{3}}\left\{2\left(G^{\prime \prime}-\frac{1}{q} G^{\prime}\right)+\left(E^{\prime \prime}-\frac{1}{q} E^{\prime}\right)\right\} X$

$R_{20}(q)=t_{r} \frac{6}{q^{4}}\left\{2\left(G^{\prime \prime}-\frac{1}{q} G^{\prime}\right)+\left(E^{\prime \prime}-\frac{1}{q} E^{\prime}\right)\right\} X$

with $v_{s}=\beta_{o}, v_{r}=\beta_{o} / \alpha_{o}, q_{1}=q_{o}$ and $q_{2}=q_{o o}$. The values of $q_{1}$ and $q_{2}$ are restricted to satisfy $c+\xi \leq\left|q_{j} \hat{\mathbf{q}}-\mathbf{c}\right| \leq c+\xi+\Delta \xi ; \quad(j=1,2)$.

The above expressions for $R_{i j}$ are independent of function $F(q)$ and its derivatives which indicates the immunity of rotation to $P$ wave velocity inhomogeneity. The role of $\delta \beta$ in $D_{i j}$ is represented by the derivatives of $G(q)$. So, in the absence of $P$ wave velocity perturbation, the dilatation of scattered near-field is controlled only by the derivatives of $G(q)$. Similar results are obtained by Leary (1995) for the scattering of far-field shear motion. $D_{2}(q)$ and $R_{2}(q)$ vanish when the perturbation in rigidity (i.e., $\rho \beta^{2}$ ) is of the form $C_{1} q^{2}+C_{2}$, where, $C_{1}$ and $C_{2}$ are constants. The strains in equations (10)-(11), then, reduce to the integrals over solid angle only.

\subsection{Special cases}

- The back scattering expressions are obtained by substituting $r=0$ in the respective equations. Thus, the back scattering strains are expressed as follows.

$$
\begin{aligned}
\nabla \cdot \delta \mathbf{u}= & u_{o} s_{o}^{2} \alpha_{o}\left[\frac { v _ { 2 } q _ { 2 } } { \beta _ { o } ^ { 2 } } \left\{\left(D_{12}\left(q_{2}\right)+c^{2} D_{10}\left(q_{2}\right)\right)\right.\right. \\
& \left.\times \int \chi_{f} d \Omega-2 c D_{11}\left(q_{2}\right) \int \chi_{f} \chi_{c} d \Omega\right\} \\
& -\frac{v_{1} q_{1}}{\alpha_{o}^{2}}\left\{\left(D_{12}\left(q_{1}\right)+c^{2} D_{10}\left(q_{1}\right)\right)\right. \\
& \left.\times \int_{\chi_{f}} d \Omega-2 c D_{11}\left(q_{1}\right) \int \chi_{f} \chi_{c} d \Omega\right\} \\
& +\int_{q_{1}}^{q_{2}}\left\{D_{22}(q)+c^{2} D_{20}(q)\right\} d q
\end{aligned}
$$

$$
\begin{aligned}
& \times \int \chi_{f} d \Omega-2 c \int_{q_{1}}^{q_{2}} D_{21}(q) d q \\
& \left.\times \int \chi_{f} \chi_{c} d \Omega\right]
\end{aligned}
$$$$
\nabla \wedge \delta \mathbf{u}=u_{o} s_{o}^{2} \alpha_{o}\left[\frac { v _ { 2 } q _ { 2 } } { \beta _ { o } ^ { 2 } } \left\{\left(R_{12}\left(q_{2}\right)+c^{2} R_{10}\left(q_{2}\right)\right)\right.\right.
$$$$
\left.\times \int \hat{\Psi}_{f} d \Omega-2 c R_{11}\left(q_{2}\right) \int \hat{\Psi}_{f} \chi_{c} d \Omega\right\}
$$$$
-\frac{v_{1} q_{1}}{\alpha_{o}^{2}}\left\{\left(R_{12}\left(q_{1}\right)+c^{2} R_{10}\left(q_{1}\right)\right)\right.
$$$$
\left.\times \int \hat{\Psi}_{f} d \Omega-2 c R_{11}\left(q_{1}\right) \int \hat{\Psi}_{f} \chi_{c} d \Omega\right\}
$$$$
+\int_{q_{1}}^{q_{2}}\left\{R_{22}(q)+c^{2} R_{20}(q)\right\} d q \int \hat{\Psi}_{f} d \Omega
$$$$
\left.-2 c \int_{q_{1}}^{q_{2}} R_{21}(q) d q \int \hat{\Psi}_{f} \chi_{c} d \Omega\right],
$$

where, $v_{1}=\left|\frac{1}{\alpha_{o}}+\frac{1}{v_{s}}\right|^{-1}, v_{2}=\left|\frac{1}{\beta_{o}}+\frac{1}{v_{s}}\right|^{-1} ; q_{o}=$ $t v_{s} /\left(1+v_{r}\right), q_{o o}=.5 t v_{s} ; t_{d}=t-q / \alpha_{o}, t_{r}=$ $t-q / \beta_{o} ; X=1 / q, X_{1}=-1 / q^{2}, X_{2}=2 / q^{3}, X_{3}=$ $-6 / q^{4}$. Above integrals are simple to be solved analytically for a given spherical shell inhomogeneity. The coefficients of $F(q)$ and $G(q)$ in $D_{11}(q)$ and $R_{11}(q)$, respectively, vanish. This implies that the role of $F(q)$ and $G(q)$ (except its derivatives) in scattering is independent of $\chi_{c}$. This, further implies that direction of $\mathbf{c}$ is not important when $\mathrm{S}$ wave velocity inhomogeneity is smooth.

- $c=0$ represents the situation when inhomogeneity is spherical around source. The expressions for strains, then reduce to

$$
\begin{aligned}
\nabla \cdot \delta \mathbf{u}= & u_{o} s_{o}^{2} \alpha_{o} \int \chi_{f}\left[\frac{v_{2} q_{2}}{\beta_{o}^{2}} D_{12}\left(q_{2}\right)\right. \\
& \left.-\frac{v_{1} q_{1}}{\alpha_{o}^{2}} D_{12}\left(q_{1}\right)+\int_{q_{1}}^{q_{2}} D_{22}(q) d q\right] d \Omega \\
\nabla \wedge \delta \mathbf{u}= & u_{o} s_{o}^{2} \alpha_{o} \int \hat{\Psi}_{f}\left[\frac{v_{2} q_{2}}{\beta_{o}^{2}} R_{12}\left(q_{2}\right)\right. \\
& \left.-\frac{v_{1} q_{1}}{\alpha_{o}^{2}} R_{12}\left(q_{1}\right)+\int_{q_{1}}^{q_{2}} R_{22}(q) d q\right] d \Omega .
\end{aligned}
$$


In this case, the strain expressions for back scattering $(r=c=0)$, further reduce to

$$
\begin{aligned}
\nabla \cdot \delta \mathbf{u}= & u_{o} s_{o}^{2} \alpha_{o}\left[\frac{v_{2} q_{2}}{\beta_{o}^{2}} D_{12}\left(q_{2}\right)-\frac{v_{1} q_{1}}{\alpha_{o}^{2}} D_{12}\left(q_{1}\right)\right. \\
& \left.+\int_{q_{1}}^{q_{2}} D_{22}(q) d q\right] \int \chi_{f} d \Omega \\
\nabla \wedge \delta \mathbf{u}= & u_{o} s_{o}^{2} \alpha_{o}\left[\frac{v_{2} q_{2}}{\beta_{o}^{2}} R_{12}\left(q_{2}\right)-\frac{v_{1} q_{1}}{\alpha_{o}^{2}} R_{12}\left(q_{1}\right)\right. \\
& \left.+\int_{q_{1}}^{q_{2}} R_{22}(q) d q\right] \int \hat{\Psi}_{f} d \Omega
\end{aligned}
$$

- Smooth variations of density inhomogeneity (i.e, $\left.E^{\prime}(q)=0\right)$ may be a little compromise but it reduces the expressions of $D_{i j}, R_{i j} ;(i=1,2 ; j=$ $0,1,2)$, to a great extent.

\section{Numerical results and discussion}

The author regrets not finding a parallel study to check the correctness of the expressions derived. The effects of various parameters on the scattering may be computed numerically. Getting these effects on expected lines, only, can assure the correctness of the results obtained. The purpose of numerical computation of scattered strains, given by integral expression (equations 10 and 11), may be to explore their use in the simulation of the records of near-fault observations. Non-availability of relevant data for a physical model compelled us to compute the strains for a hypothetical model. Hypothetical model is constructed keeping in mind the uniwell/crosswell survey for time-gap monitoring of oil-water barrier approaching the production well in a reservoir. It can, also, be a mathematical model for monitoring the dynamism around a known active fault.

In the model considered, the density perturbation is assumed constant, only to simplify the computation. Perturbations in wave velocities are defined by $F(q)=G(q)=0.1 \sin \{.1(q-\xi)\}$. For simplicity, in the spherical co-ordinate system centred at $C$, the source is placed along the polar axis at a distance $c$ from $C$ and at radial distance $\xi$ from inner edge of the shell. Inhomogeneity is a part of spherical shell (thickness $\Delta \xi$ ) with arc length $8 \mathrm{~m}$ in the polar plane and arc length of $5 \mathrm{~m}$ in the azimuthal plane. Polar axis is assumed to be passing through the centre of the curved face of the inhomogeneity. The polar angle subtended by the inhomogeneity at $C$ is then approximated as $8 /(c+\xi+.5 \Delta \xi)$ and azimuthal angle is
$5 /(c+\xi+.5 \Delta \xi)$. Direction of impulsive force $\hat{\mathbf{n}}=$ $(1,1,1) / \sqrt{3}$. Receiver is located at distance $r$ from source in the direction $(1,1,0) / \sqrt{2}$. Wave velocities are assumed as $\alpha_{o}=4 \mathrm{~km} / \mathrm{sec} ; \beta_{o}=2.5 \mathrm{~km} / \mathrm{sec}$. Integrals over $d q$ and $d \Omega(=d \chi d \phi)$, are computed through summation method. To study the effects of size and shape of inhomogeneity, its distance from source and receiver, the strains are computed for hypothetical models and are exhibited in figure 2 to figure 5 .

Dilatation and rotation components are plotted, in figure 2, for values of $\xi=4,4.5,5 \mathrm{~m} . \Delta \xi=$ $4 \mathrm{~m}, r=6 \mathrm{~m}$, and $c=10 \mathrm{~m}$. The strains decrease, very rapidly, with the increase of $\xi$. Even half a meter increase in $\xi$ may reduce the scattering by one half. Rate of decrease of dilatation and rotation is almost similar. Time of travel also increases with $\xi$, as expected.

The variations in scattering with the thickness of shell are exhibited in figure 3 , for values of $\Delta \xi=3,5,10 \mathrm{~m}$. Other parameters remain same and $\xi=4 \mathrm{~m}$. The effect on dilatation is not significant and resultant rotation increases slightly with the increase of thickness. However, in the model considered the z-rotation is decreasing with the increase of $\xi$.

Figure 4 presents the effect of source receiver distance on the strains for the values of $r=0,3,8 \mathrm{~m}$. To compare with the back scattering $(r=0)$ case the receiver is placed on polar axis, i.e., $\hat{\mathbf{r}}=$ $(0,0,-1) . \Delta \xi=4 \mathrm{~m}$. The dilatation and rotation decrease rapidly with the increase of $r$.

The variations of strains with the change in the curvature of the inhomogeneous spherical shell, are plotted in figure 5 , for the values of $c=-1,0,2,20$ on meter scale. The corresponding values of curvature $\{=1 /(c+\xi)\}$ are $0.33,0.25,0.17,0.04 \mathrm{~m}^{-1}$, when $\xi=4 \mathrm{~m}$. $\hat{\mathbf{r}}=(1,1,0) / \sqrt{2}$ and $r=6 \mathrm{~m}$. Both dilatation and rotation decrease with decrease of curvature. This decrease has larger rate for dilatation as compared to rotation. The effect of curvature change on scattering is not significant for larger values of c (e.g., $c \geq 10)$.

\section{Conclusions}

From the analytical expressions and special cases, the following conclusions may be drawn:

(i) The rotation of scattering from a radial inhomogeneity, are independent of $P$ wave velocity inhomogeneity. This implies that any observed effect of $P$ wave velocity inhomogeneity on scattered rotation must be due to the spherical asymmetry.

(ii) The dilatation of scattering from a radial inhomogeneity, does not depend upon $S$ 

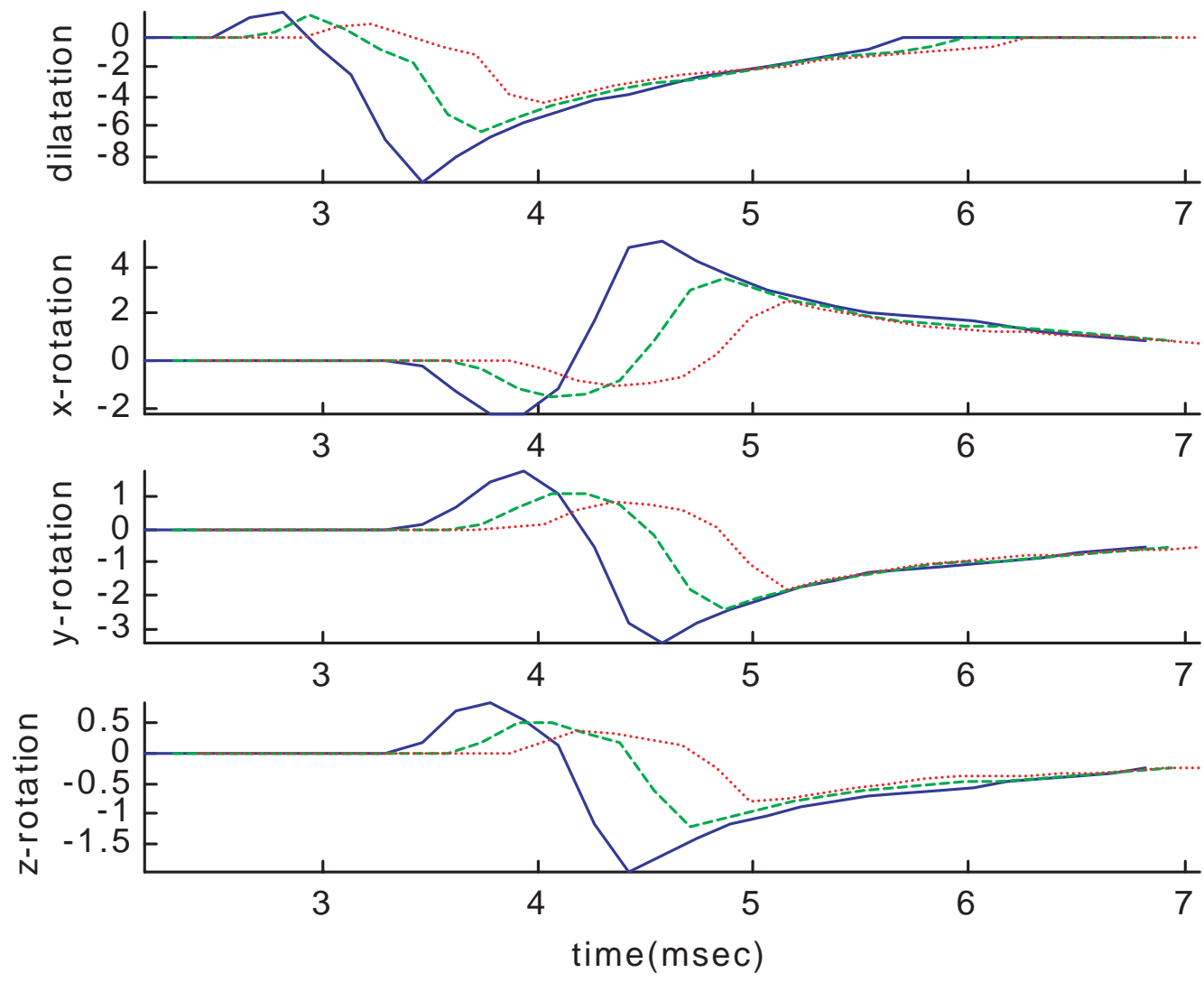

Figure 2. Variations in scattering strains with the source-inhomogeneity distance $\{\xi=4 \mathrm{~m}:-; \xi=4.5 \mathrm{~m}:-----; \xi=5 \mathrm{~m}: \cdots \cdots \cdots \cdots\}$.
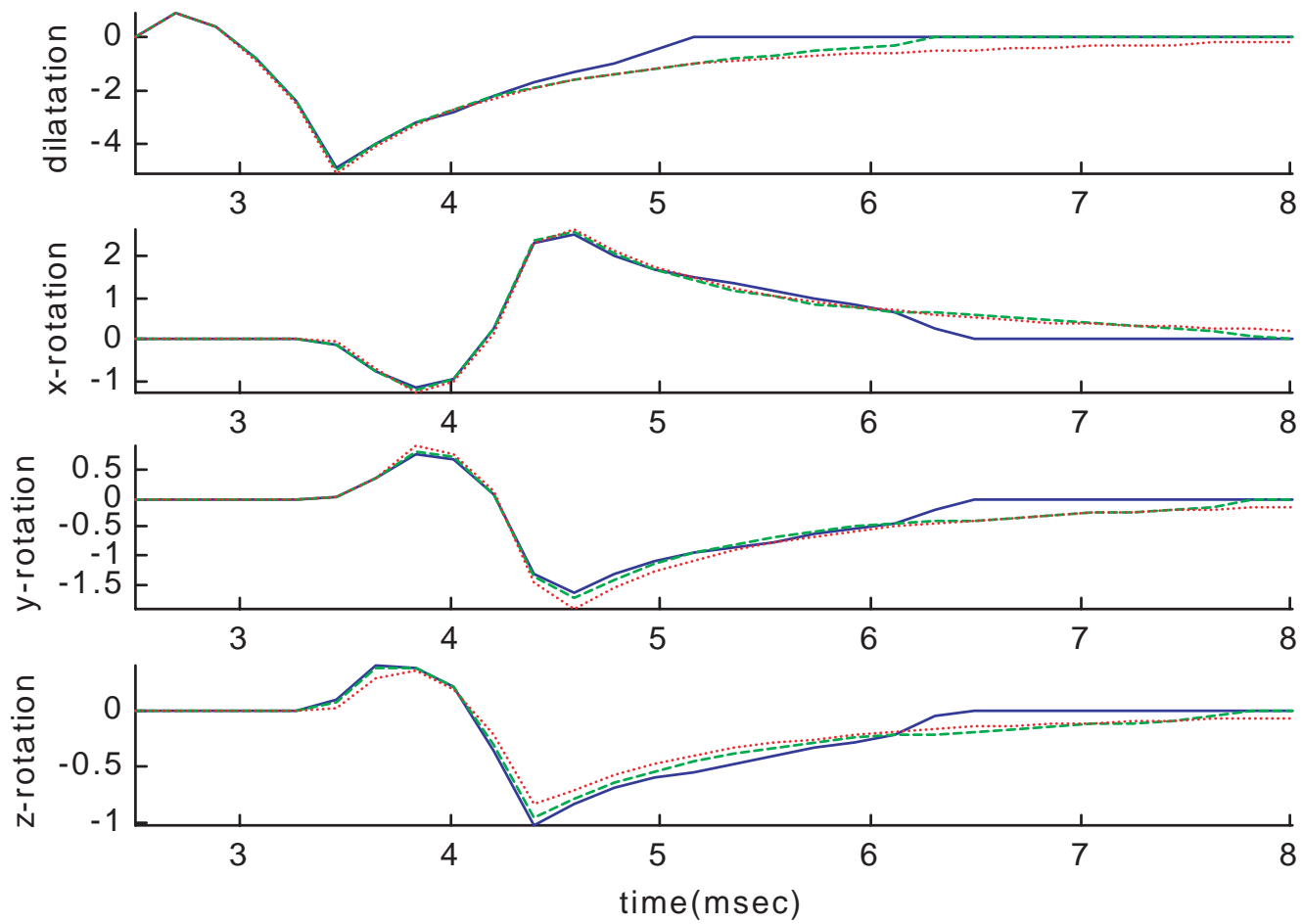

Figure 3. Variations in scattering strains with the inhomogeneity thickness $\{\Delta \xi=3 \mathrm{~m}: \ldots \ldots$ _ $; \Delta \xi=5 \mathrm{~m}:-----; \Delta \xi=10 \mathrm{~m}: \cdots \cdots \cdots \cdots\}$. 

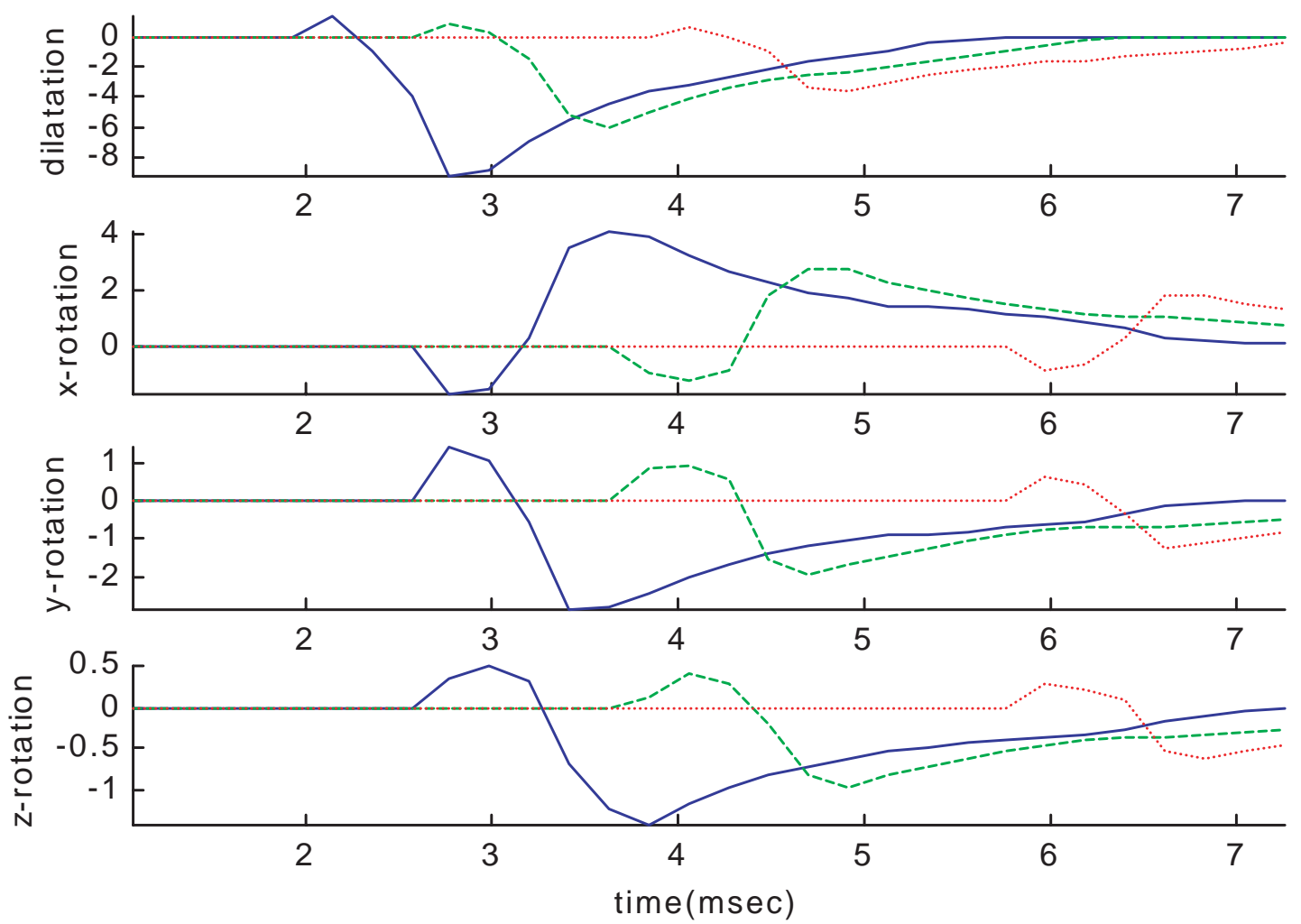

Figure 4. Variations in scattering strains with the source-receiver distance $\{r=0$ : $; r=3 \mathrm{~m}:-----; r=8 \mathrm{~m}: \cdots \cdots \cdots \cdots\}$.
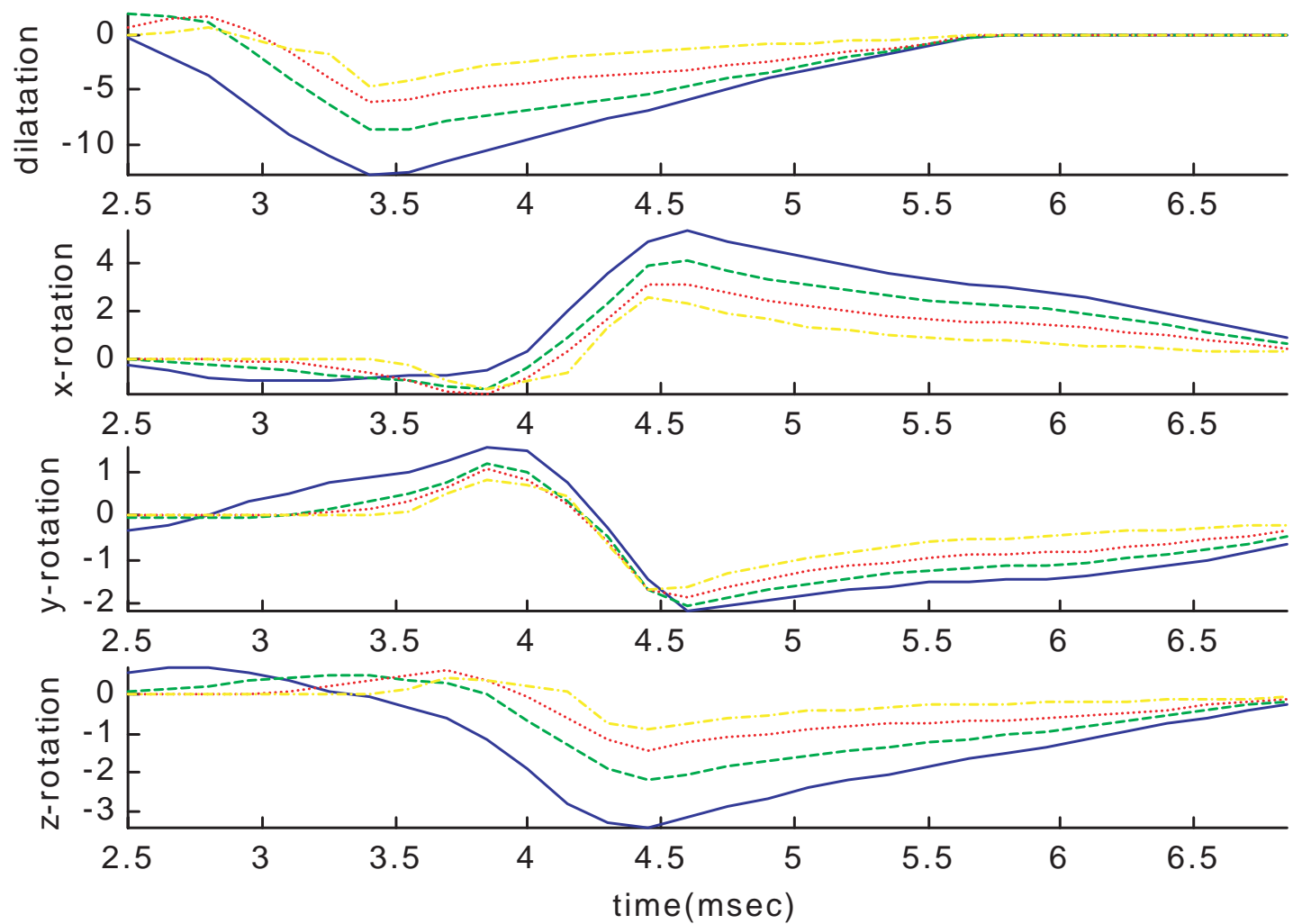

Figure 5. Variations in scattering strains with the inhomogeneity curvature $\{c=-1: \ldots ; c=0:-----; c=2 \mathrm{~m}: \cdots \cdots \cdots+\ldots ; c=20 \mathrm{~m}:-\cdot-\cdot-\cdot-\}$. 
wave velocity inhomogeneity but its gradient. Hence, the inhomogeneity with smooth perturbation of shear wave velocity does not affect the $P$ motion in scattered near-field.

(iii) Combining conclusions (i) and (ii) implies that, in the absence of $P$ wave velocity perturbation, the dilatation of scattered near-field is controlled only by the derivatives of $G(q)$.

(iv) No dilatation of scattering for incidence in the direction normal to the direction of impulsive force (due to the presence of $\chi_{f}$ in equation (6)).

(v) The presence of $\hat{\Psi}_{f}$ in equation (7) means no rotation of scattering for incidence along the direction of impulsive force.

(vi) Direction of centre of curvature from source is not important for back scattering when $S$ wave velocity inhomogeneity is smooth.

(vii) For back scattering from a complete spherical shell or from an azimuthal ring, strains of scattering vanishes when source is placed at the centre of curvature (i.e., $c=r=0$ ).

(viii) The scattering of near-field does not depend upon the differential of $P$ wave velocity inhomogeneity.

The numerical results are obtained for a very particular hypothetical model and hence may not qualify for generalisation but these may provide some useful information for simulation studies. The few observations made out of the discussion of these results are as follows:

- The strains decrease drastically with the increase of distance of source from inhomogeneity.

- Both dilatation and rotation of near-field scattering decrease rapidly with the increase of distance between source and receiver.

- Change of shape of inhomogeneity from spherical to rectangular decreases the strains of near-field scattering.

- As compared to rotation, the dilatation of nearfield scattering is more sensitive to the shape of inhomogeneity.

This was an attempt to study the strains of three dimensional scattering from a spherical inhomogeneity in time domain. The time domain scenario always presents a more transparent picture of any process. A specific use of this study may be in the numerical simulation of time-lapse borehole seismic survey data aimed at monitoring the oil-water interface in flow heterogeneous reservoirs. Time lapse tracking of oil-water substitution and monitoring reservoir stress conditions help to yield spatially well-constrained reservoir models. The simulation of ground motion time series at a specific site due to an earthquake is the best kind of information a seismologist can provide for engineering structural design (Yu 1994). Borehole recording of ground motion is used to study the subsidence of ground and non-linear behaviour of soil (Iida 2000). Earthquake engineers may be interested in the realistic ground tilt/subsidence that are site specific. Hence, this study may be useful in the predictive studies of seismic hazards due to an impending earthquake in any region. Engineers working on the response of stressed materials may also find some interest in this study. Checking unwanted inhomogeneities and flaws through the non-destructive testing of materials may be another application of this study.

\section{References}

Aki K and Richards P G 1980 Quantitative Seismology, Theory and Methods, Vol. I \& II, (New York: Freeman)

Gritto R, Korneev V A and Johnson L R 1995 Low frequency elastic wave scattering by an inclusion: limits of applications; Geophys. J. Int. $120677-692$

Gurevich B, Sadovnichaja A P, Lopatnikov S L and Shapiro S A 1992 The Born approximation in the problem of elastic wave scattering by a spherical inhomogeneity in a fluid saturated porous media; Appl. Phys. Lett. 61 1275-1277

Gurevich B, Sadovnichaja A P, Lopatnikov S L and Shapiro S A 1998 Scattering of a compressional wave in a poroelastic medium by an ellipsoidal inclusion; Geophys. J. Int. 133 91-103

Iida M 2000 A systematic method for analysing borehole recording to estimate the wave field in the lakebed zone of Mexico City; Bull. Seism. Soc. Am. 90 126880

Korneev V A and Johnson L R 1993 Scattering of elastic waves by a spherical inclusion-I: Theory and numerical results; Geophys. J. Int. 115 230-250

Leary P C 1990 Basement rock fracture structure from Cajon Pass, California and Siljan Ring, Sweden, borehole geophysical logs, Expanded Abstracts; 60th Ann. Int. Meet., 153-55

Leary P C 1995 Quantifying crustal fracture heterogeneity by seismic scattering; Geophys. J. Int. 122 125142

Leary P C and Abercrombie R 1994 Frequency dependent crustal scattering and absorption at $5-160 \mathrm{~Hz}$ from coda decay observed at $2.5 \mathrm{~km}$ depth; Geophys. Res. Lett. 21 1683-86

Rikitake T (ed) 1981 Current Research in Earthquake Prediction, I. Developments in Earth and Planetary Sciences (London: D. Reidel Publishing Co.)

Rose J H 1989 Elastic wave inverse scattering in nondestructive evaluation; PAGEOPH 131 715-739

Sato H 1984 Attenuation and envelope formation of three component seismograms of small local earthquakes in randomly inhomogeneous lithosphere; J. Geophys. Res. 89 1221-1242

Sharma M D 2001 Scattering of a spherical pulse from a small inhomogeneity: Dilatation and rotation; Proc. Indian Acad. Sci. (Earth Planet. Sci.) 110(3) $1-9$

Wu R S 1989 The perturbation method in elastic wave scattering; PAGEOPH 131 605-637

Wu R S and Aki K 1985a Scattering characteristics of elastic waves by an elastic heterogeneity; Geophysics $\mathbf{5 0}$ $582-595$ 
Wu R S and Aki K 1985b Elastic wave scattering by a random medium and the small scale inhomogeneities in the lithosphere; J. Geophys. Res. 90 10261-10273

Wu R S and Aki K 1988 Introduction: Seismic wave scattering in three-dimensionally heterogeneous earth; PAGEOPH 128 1-6
Yu G 1994 Some aspects of earthquake seismology; slip partitioning along major convergent plate boundaries: Composite source model for estimation of strong ground motion and non-linear soil response modelling; Ph.D. Thesis, University of Nevada, USA, $144 \mathrm{p}$.

MS received 2 April 2002; revised 11 March 2003 